\title{
PDGF-BB promotes the differentiation and proliferation of MC3T3-E1 cells through the Src/JAK2 signaling pathway
}

\author{
QI LIU $^{1 *}, \mathrm{YUNFENG} \mathrm{ZHOU}^{1 *}$ and ZUBING $\mathrm{LI}^{2}$ \\ ${ }^{1}$ The State Key Laboratory Breeding Base of Basic Science of Stomatology (Hubei-MOST) and \\ Key Laboratory of Oral Biomedicine Ministry of Education; ${ }^{2}$ Department of Oral and Maxillofacial Surgery, \\ School and Hospital of Stomatology, Wuhan University, Wuhan, Hubei 430079, P.R. China
}

Received November 2, 2017; Accepted July 20, 2018

DOI: $10.3892 / \mathrm{mmr} .2018 .9351$

\begin{abstract}
Platelet-derived growth factor-BB (PDGF-BB) serves a critical function in human osteoblast differentiation and proliferation. Src and Janus kinase 2 (JAK2) are involved in these processes. In our previous study, it was identified that Src could promote the phosphorylation of JAK2. However, it has yet to be determined whether the Src/JAK2 signaling pathway affects PDGF-BB-mediated osteoblast differentiation and proliferation. In the present study, western blotting, polymerase chain reaction, alizarin red staining, alkaline phosphatase and Cell Counting kit- 8 were employed to explore these questions. Firstly, it was demonstrated that PDGF-BB activates the Src/JAK2 signaling pathway in MC3T3-E1 cells in a time-dependent manner. Furthermore, it was demonstrated that PDGF-BB expression promoted MC3T3-E1 cell differentiation and proliferation; this process was suppressed by AG1295, SU6656 and AG490, which are inhibitors of PDGFR- $\beta$, Src and JAK2, respectively. SU6656 downregulated the activity of Src and JAK2, while AG490 only downregulated JAK2 activity. Therefore, it was concluded that Src is upstream of JAK2. PDGF-BB also upregulated the expression of osteogenesis-associated genes, and the formation of mineral nodules. However, these effects were markedly inhibited by treatment with SU6656. This indicated that PDGF-BB promoted MC3T3-E1 cell differentiation and proliferation by activating the Src/JAK2 signaling pathway. These results
\end{abstract}

Correspondence to: Professor Zubing Li, Department of Oral and Maxillofacial Surgery, School and Hospital of Stomatology, Wuhan University, 237 Luoyu Road, Wuhan, Hubei 430079, P.R. China

E-mail: lizubing@whu.edu.cn

"Contributed equally

Abbreviations: PDGF-BB, platelet-derived growth factor-BB PDGFR $\beta$, platelet-derived growth factor-BB receptor $\beta$; JAK2, Janus kinase 2; STAT, signal transducers and activators of transcription; CCK8, Cell Counting kit-8

Key words: PDGF-BB, Src, JAK2, MC3T3-E1 cells, differentiation, proliferation suggested that PDGF-BB may have potential applications in the treatment of osteoporosis and bone fractures.

\section{Introduction}

The skeletal system is one of the most important systems in the human body. It serves as the body's structural support, provides a framework for attachment of tissues, protects vital organs and helps direct the forces necessary for movement $(1,2)$. Bone homeostasis depends on a dynamic balance between osteoblasts and osteoclasts (2). An imbalance in bone homeostasis leads to metabolic diseases such as osteoporosis, osteopetrosis, and Paget's diseases (3). Therefore, the components and mechanisms of bone homeostasis have been widely investigated. Osteoblast proliferation and differentiation are important in maintaining bone metabolic balance, and these processes are regulated by numerous signals and pathways.

Platelet-derived growth factor-BB (PDGF-BB) can promote the proliferation and differentiation of human osteoblasts (4), and stimulate the synthesis of interleukin-6 in osteoblasts (5), thus promoting bone formation and regeneration (6). Following the binding of PDGF-BB to its receptor (PDGFR- $\beta$ ), endogenous tyrosine phosphorylating activity and intercellular signaling are activated (7). PDGF-BB secreted by preosteoclasts promotes bone formation through stimulating the migration and angiogenesis of endothelial progenitor cells and MSCs (8).

Src interacts with PDGFR through its Src homology 2, coiled-coil and FCH (Fer/Fes/Fps/Cip4 homology) domains (9). Src not only serves key functions in physiological and pathological processes, including cell survival, cell differentiation, inflammation (10), tumorigenesis, invasion and metastasis (11), but it also serves a critical function in bone metabolism. Although western blot analysis has indicated that PDGF-BB induces the phosphorylation of Src and Janus kinase 2 (JAK2) in pancreatic stellate cells in a time-dependent manner (7), the interaction between Src and PDGF-BB in osteoblast cells is poorly understood.

The JAK family consists of four members, JAK1, JAK2, JAK3 and Tyk2, which are activated by cytokines binding their receptor. This activation leads to the subsequent phosphorylation and activation of signal transducer and activation of transcription (STAT) transcription factors $(3,12)$. JAK2 was 
previously demonstrated to be associated with MC3T3-E1 cell proliferation in conditioned media from mouse osteosarcoma cells (13).

The aim of the present study was to investigate the complex associations between PDGF-BB, Src and JAK2, and to investigate their interaction in MC3T3-E1 cells.

\section{Materials and methods}

Reagents. Antibodies against phosphorylated (p-)PDGFR $\beta$ (cat. no. 3161), PDGFR $\beta$ (cat. no. 3175), p-Src (cat. no. 2105), Src (cat. no. 2110), p-JAK2 (cat. no. 3771), JAK2 (cat. no. 3230; all dilution, 1:1,000), GAPDH (cat. no. 5174), $\beta$-actin (cat. no. 8457; both dilution, 1:2,500) and Runt-related transcription factor 2 (RUNX2; cat. no. 98059; dilution, 1:2,000), were purchased from Cell Signaling Technology, Inc. (Danvers, MA, USA). The OSTERIX (cat. no. ab22552) and anti-COL1 $\alpha 1$ (cat. no. ab166606; both dilution, 1:2,000) antibodies were purchased from Abcam (Cambridge, UK). Dexamethasone, ascorbic acid, $\beta$-glycerophosphate, AG1295 and hexadecylpyridinium chloride monohydrate were purchased from Sigma-Aldrich (Merck KGaA, Darmstadt, Germany). SU6656 and AG490 were purchased from Calbiochem (Merck KGaA). PDGF-BB was purchased from Peprotech, Inc. (Rocky Hill, NJ, USA).

MC3T3-E1 cell culture. Murine osteoblast-like MC3T3-E1 cells (China Center for Type Culture Collection, Wuhan, China) were cultured in $\alpha$-MEM complete medium supplemented with $10 \%$ fetal bovine serum (FBS) and $1 \%$ penicillin-streptomycin, at $37^{\circ} \mathrm{C}$, in a humidified atmosphere of $95 \%$ air and $5 \% \mathrm{CO}_{2}$. The cell culture medium was changed every 2-3 days. Cells were divided into five groups, including bovine serum albumin (BSA), PDGF-BB, PDGF-BB +20 $\mu \mathrm{mol} / 1$ AG1295, $\mathrm{PDGF}-\mathrm{BB}+2 \mu \mathrm{mol} / 1 \mathrm{SU} 6656$ and PDGF-BB $+10 \mu \mathrm{mol} / 1$ AG490 $10 \mu \mathrm{mol} / \mathrm{l})$. All PDGF-BB used in this research was $25 \mathrm{ng} / \mathrm{ml}$.

Western blot analysis. The MC3T3-E1 cells were cultured in a 6-well plate, grown to $70-80 \%$ confluence, and then starved in serum-free medium for $24 \mathrm{~h}$. The cells were lysed in radioimmunoprecipitation assay (RIPA) lysis buffer (Sigma-Aldrich; Merck $\mathrm{KGaA}$ ) at $4^{\circ} \mathrm{C}$ for $30 \mathrm{~min}$. Equal amounts of protein quantified by BCA $(60 \mu \mathrm{g})$ were loaded onto $10 \%$ SDS-polyacrylamide gels. Following electrophoresis, proteins were transferred to polyvinylidene fluoride membranes (EMD Millipore, Billerica, MA, USA). Blots were blocked with 5\% skimmed milk (Difco; BD Biosciences, Franklin Lakes, NJ, USA) at room temperature for $1 \mathrm{~h}$, then incubated with the aforementioned primary antibodies overnight at $4^{\circ} \mathrm{C}$. Afterwards, the blots were washed and incubated with horseradish peroxidase-conjugated secondary antibodies (Beijing TDY Biotech Co., Ltd., Beijing China; dilution, 1:5,000) for $2 \mathrm{~h}$. Blots were detected using an ECL kit (GE Healthcare, Chicago, IL, USA) according to the manufacturer's protocol. Western blot bands were quantified by densitometric analysis using Photoshop CS6 (Adobe Systems, Inc., San Jose, CA, USA).

Osteoblast differentiation. Murine osteoblast-like MC3T3-E1 cells were cultured at $5 \times 10^{4}$ cells $/ \mathrm{cm}^{2}$ in osteogenic medium containing $10 \% \mathrm{FBS}, 0.1 \mu \mathrm{M}$ dexamethasone, $10 \mathrm{mM} \beta$-glycerophosphate and $50 \mu \mathrm{g} / \mathrm{ml}$ ascorbic acid for 21 days. At days 3 and 7, cells were harvested for reverse transcription-quantitative polymerase chain reaction (RT-qPCR) analysis or western blotting. Cells were fixed and alkaline phosphatase (ALP) staining was performed at days 3 and 7, whereas Alizarin Red staining was performed at days 14 and 21 .

$R N A$ isolation and RT-qPCR. Total RNA was extracted from cells with TRIzol reagent (Invitrogen; Thermo Fisher Scientific, Inc.) according to the manufacturer's protocol. Total RNA $(1 \mu \mathrm{g})$ was used for cDNA synthesis using the RevertAid First Strand cDNA Synthesis kit (Eppendorf, Hamburg, Germany). qPCR was performed in triplicate using SYBR Green Master mix (Takara Biotechnology Co., Ltd., Dalian, China) on an Applied Biosystems 7500 Real-Time PCR system (Thermo Fisher Scientific, Inc.) as follows: $95^{\circ} \mathrm{C}$ for $30 \mathrm{sec}$, followed by 40 cycles of $95^{\circ} \mathrm{C}$ for $5 \mathrm{sec}, 60^{\circ} \mathrm{C}$ for $31 \mathrm{sec}$ and $95^{\circ} \mathrm{C}$ for $15 \mathrm{sec}$, then $60^{\circ} \mathrm{C}$ for $1 \mathrm{~min}$ and $95^{\circ} \mathrm{C}$ for $15 \mathrm{sec}$. The qPCR results were automatically analyzed using the Applied Biosystems 7500 system. The $2^{-\Delta \Delta \mathrm{Cq}}$ method (14) was used to calculate the relative gene expression level. The primers used are presented in Table I.

ALP staining. Cells were washed twice with PBS, fixed with $4 \%$ paraformaldehydefor $10 \mathrm{~min}$,rinsed withdeionized waterand stained with a 5-bromo-4-chloro-3'-indolyphosphate/nitro-blue tetrazolium ALP color development kit (Beyotime Institute of Biotechnology, Haimen, China) for $12 \mathrm{~h}$ away from direct light, according to the manufacturer's protocol. Images were subsequently obtained.

Alizarin Red staining and mineralization assay. Cells were washed twice with cold PBS and fixed with $4 \%$ paraformaldehyde for $10 \mathrm{~min}$. They were then stained with $50 \mathrm{mM}$ Alizarin Red $\mathrm{S}$ ( $\mathrm{pH} 4.2$ ) for $10 \mathrm{~min}$ at room temperature, and images were obtained. In order to determine the extent of calcium deposition, the mineralization of calcium nodules was quantified. After staining, the cells were washed three times with PBS. Hexadecylpyridinium chloride monohydrate (10\%) was added and incubated for $20 \mathrm{~min}$ at room temperature. The absorbance of the supernatant was measured at $562 \mathrm{~nm}$ in triplicate using a Multiskan EX plate reader (Power Wave $\mathrm{XS}_{2}$; Thermo Fisher Scientific, Inc.). Finally, cells were washed in PBS and lysed with RIPA buffer. and protein content quantified by BCA and calcium levels were normalized to the total protein content.

Cell proliferation assay. Proliferation activity was measured using a Cell Counting kit-8 (CCK-8; Dojindo Molecular Technologies, Inc., Kumamoto, Japan) assay. MC3T3-E1 cells were seeded into a 96 -well plate at $2 \times 10^{4}$ cells $/ \mathrm{cm}^{2}$. The medium was removed following adherence, and the cells were continuously cultured for $72 \mathrm{~h}$. CCK-8 solution (10 $\mu \mathrm{l})$ was added to $100 \mu \mathrm{l}$ of medium in each well, and incubated with the cells for $2 \mathrm{~h}$, before measuring the optical density (OD) at $450 \mathrm{~nm}$. All experiments were performed in six replicates.

Statistical analysis. All quantitative data are expressed as the mean \pm standard deviation. Analysis was performed with 
Table I. Primers used for reverse transcription-quantitative polymerase chain reaction.

\begin{tabular}{lll}
\hline Gene & \multicolumn{1}{c}{ Forward $\left(5^{\prime}-3^{\prime}\right)$} & Reverse $\left(5^{\prime}-3^{\prime}\right)$ \\
\hline GAPDH & TATCGGACGCCTGGTTAC & CTGTGCCGTTGAACTTGC \\
RUNX2 & TCATTCAGTGACACCACCAGG & TGTAGGGGCTAAAGGCAAAA \\
OSTERIX & AGAAGCCATACACTGACCTTTC & GGTGGGTAGTCATTGGCATAG \\
ALP & GAGATGGTATGGGCGTCTC & GTTGGTGTTGTACGTCTTGGA \\
OCN & GACAAGTCCCACACAGCAACT & GGACATGAAGGCTTTGTCAGA \\
OPN & CCCATCTCAGAAGCAGAATCTT & GTCATGGCTTTCATTGGAGTTG \\
COL $1 \alpha 1$ & GACATGTTCAGCTTTGTGGACCTC & GGGACCCTTAGGCCATTGTGTA
\end{tabular}

ALP, alkaline phosphatase; OCN, osteocalcin; OPN, osteopontin; COL1 1 1, collagen type $1 \alpha 1$; Runx2, Runt-related transcription factor 2.

A

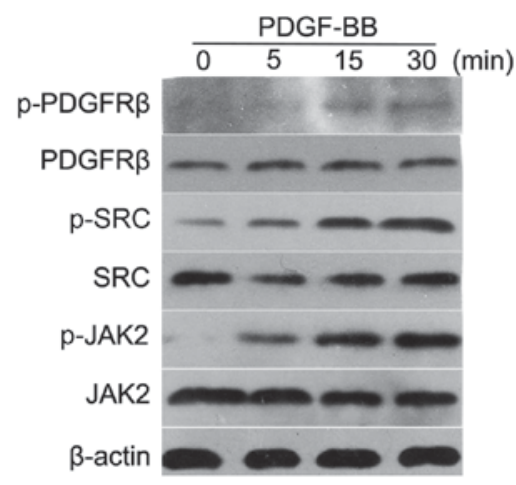

C

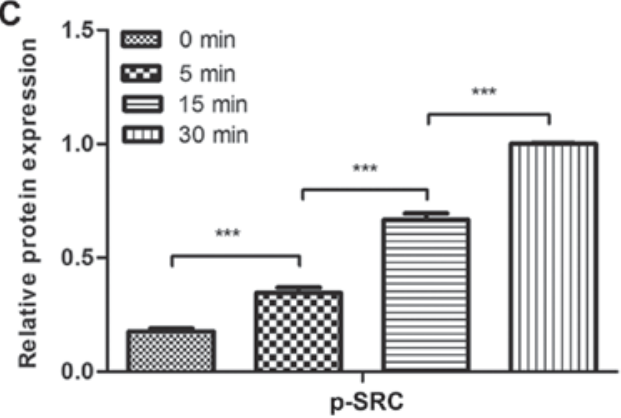

B

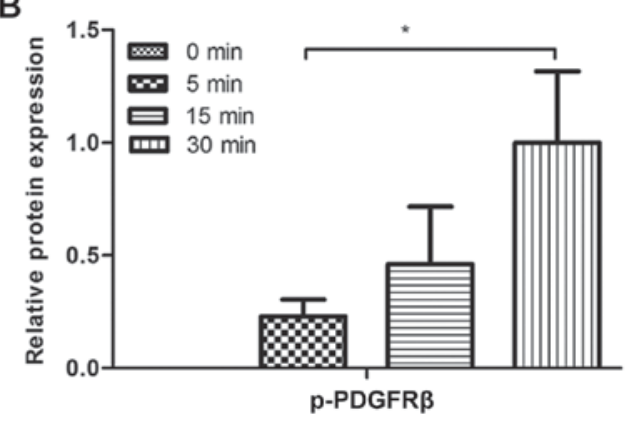

D

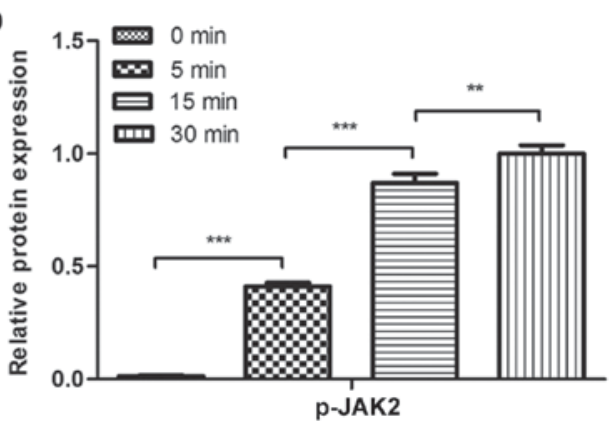

Figure 1. PDGF-BB activates PDGFR $\beta$, Src and JAK2 in MC3T3-E1 cells. (A) MC3T3-E1 cells were serum-starved overnight and treated with $25 \mathrm{ng} / \mathrm{ml}$ PDGF-BB for the indicated periods of time. Western blot analysis was performed to determine the levels of phosphorylated (B) PDGFR $\beta$, (C) Src and (D) JAK2. Densitometric analysis of the associated bands were expressed as the relative optical density of the bands, corrected using $\beta$-actin as the control (normalization band). Data are presented as the mean \pm standard deviation $(\mathrm{n}=3) .{ }^{*} \mathrm{P}<0.05,{ }^{* *} \mathrm{P}<0.01$ and ${ }^{* * *} \mathrm{P}<0.001$, as indicated. PDGF-BB, platelet-derived growth factor-BB; PDGFR $\beta$, PDGFR-BB receptor $\beta$; JAK2, Janus kinase 2; p-, phosphorylated.

GraphPad Prism 5 (GraphPad Software, Inc., La Jolla, CA, USA). Comparisons were evaluated with one-way or two-way analysis of variance followed by Tukey's post-hoc test. $\mathrm{P}<0.05$ was considered to indicate a statistically significant difference. Each experiment was repeated $\geq 3$ times, and representative images are presented.

\section{Results}

PDGF-BB activates $P D G F R \beta$, Src and JAK2 in MC3T3-E1 cells. To evaluate whether PDGF-BB could activate PDGFR $\beta$, Src and JAK2 in MC3T3-E1 cells, the cells were treated with PDGF-BB at a range of different durations, followed by the analysis of PDGFR $\beta$, Src and JAK2 phosphorylation. It was identified that PDGFR $\beta$, Src and JAK2 were rapidly phosphorylated by PDGF-BB at $5 \mathrm{~min}$ and their phosphorylation levels increased over time for the first 30 min (Fig. 1A-D). These results indicated that PDGFR $\beta$, Src and JAK2 were expressed in MC3T3-E1 cells and could be activated by PDGF-BB in a time-dependent manner within $30 \mathrm{~min}$.

Src lies upstream of JAK2 in MC3T3-E1 cells. Subsequently, it was investigated whether the phosphorylation of Src was performed directly by PDGFR $\beta$, and whether Src was an upstream molecule of JAK2, in MC3T3-E1 cells. Therefore, the effects of low molecular mass inhibitors against PDGFR $\beta$ (AG1295), Src family kinases (SU6656) and JAK2 (AG490) on Src phosphorylation were determined. Inhibition of PDGFR $\beta$ and Src kinase activity resulted in the inhibition of 
A

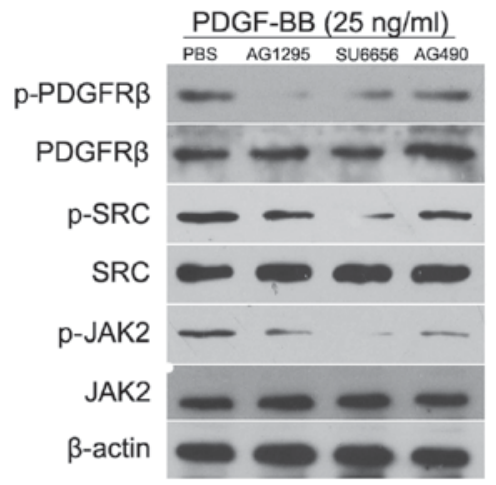

C

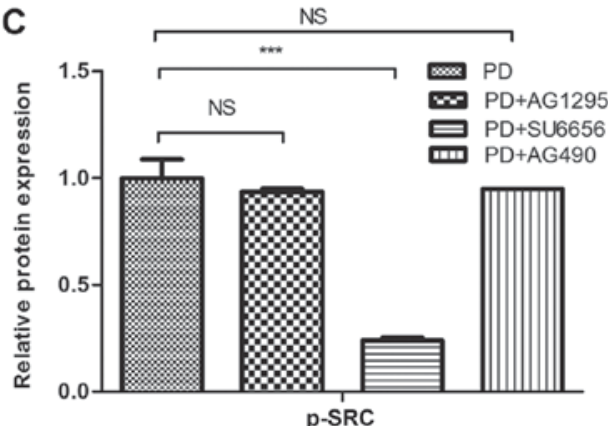

B

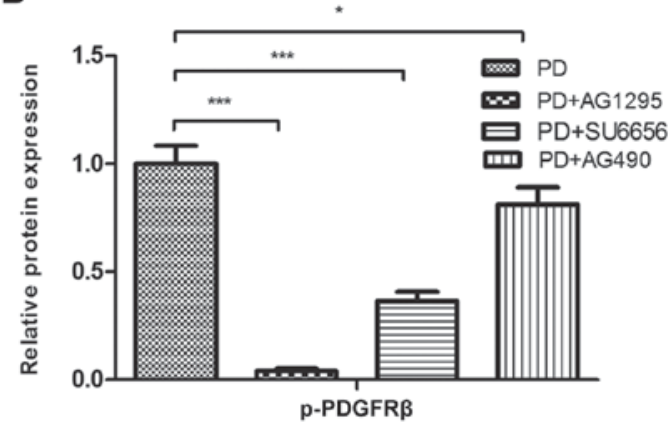

D

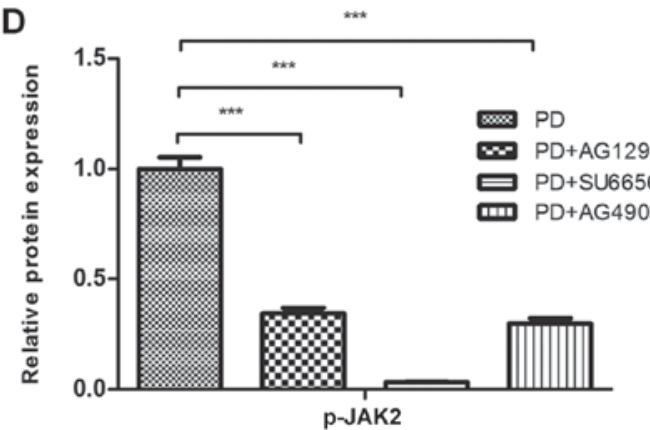

Figure 2. Src lies in the upstream of JAK2 in MC3T3-E1 cells. (A) MC3T3-E1 cells were serum-starved overnight and pretreated for 30 min with inhibitors (AG1295 $20 \mu \mathrm{mol} / 1$, SU6656 $2 \mu \mathrm{mol} / 1$ and AG490 $10 \mu \mathrm{mol} / 1$ ) as indicated, and stimulated with $25 \mathrm{ng} / \mathrm{ml}$ PDGF-BB at the same time. Densitometric analysis of the associated bands for (B) PDGFR $\beta$, (C) Src and (D) JAK2 were expressed as relative optical density of the bands, corrected using $\beta$-actin as control (normalization band). Data are presented as the mean \pm standard deviation $(\mathrm{n}=3) .{ }^{*} \mathrm{P}<0.05$ and ${ }^{* * * *} \mathrm{P}<0.001$, as indicated. PDGF-BB, platelet-derived growth factor-BB; PDGFR $\beta$, PDGFR-BB receptor $\beta$; JAK2, Janus kinase 2 ; NS, not significant; p-, phosphorylated.
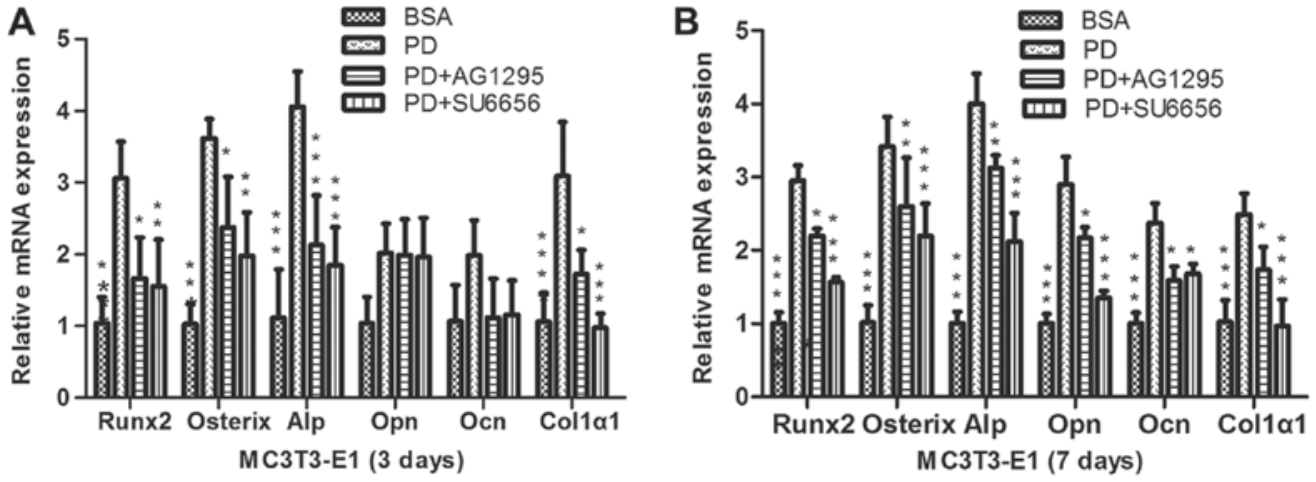

Figure 3. PDGF-BB promotes osteogenic relative gene expression via the Src/JAK2 signaling pathway. (A) MC3T3-E1 cells were treated with BSA (25 ng/ml), PDGF-BB ( $25 \mathrm{ng} / \mathrm{ml})$, PDGF-BB $(25 \mathrm{ng} / \mathrm{ml})+$ AG1295 $(20 \mu \mathrm{mol} / \mathrm{l})$ and PDGF-BB $(25 \mathrm{ng} / \mathrm{ml})+$ SU6656 $(2 \mu \mathrm{mol} / \mathrm{l})$, in osteogenic media for $3 \mathrm{days}$ or (B) for 7 days. Data are presented as the mean \pm standard deviation $(\mathrm{n}=3)$. ${ }^{*} \mathrm{P}<0.05,{ }^{* * *} \mathrm{P}<0.01$ and ${ }^{* * * *} \mathrm{P}<0.001$, vs. PD. BSA, bovine serum albumin; PDGF-BB, platelet-derived growth factor-BB; JAK2, Janus kinase 2; NS, not significant; Runx2, Runt-related transcription factor 2; Alp, alkaline phosphatase; Ocn, osteocalcin; Opn, osteopontin; Col1 $\alpha 1$, collagen type $1 \alpha 1$.

JAK2 phosphorylation (Fig. 2A and D). However, the inhibition of JAK2 activity did not affect PDGF-BB-induced Src phosphorylation (Fig. 2A and C). SU6656 and AG490 inhibited the activity of PDGFR $\beta$ (Fig. 2B), while previous study showed that AG490 had no effect on PDGFR $\beta$ (9). Therefore, we hypothesized that Src and JAK2 may be involved in other pathways that affect the phosphorylation of PDGFR $\beta$ in MC3T3-E1 cells, and that Src was upstream of JAK2 in MC3T3-E1 cells.

PDGF-BB promotes the differentiation and mineralization of MC3T3-E1 cells via the SrC/JAK2 signaling pathway. In order to evaluate whether the Src/JAK2 signaling pathway served a function in MC3T3-E1 cell differentiation and proliferation, the effects of PDGF-BB were measured in MC3T3-E1 cells cultured in osteogenic differentiation media. As identified with, the expression of osteoblast differentiation-associated genes, including Runx2, a key transcription factor (15), and Osterix, ALP and Col1 $\alpha 1$, increased in the PDGF-BB group compared with the control, PDGF-BB + AG1295 and PDGF-BB + SU6656 groups following culture in osteogenic differentiation media for 3 days (Fig. 3A). The same trend was observed for the expression of osteocalcin $(\mathrm{OCN})$ and osteopontin (OPN) at 7 days (Fig. 3B). The expression levels 
A

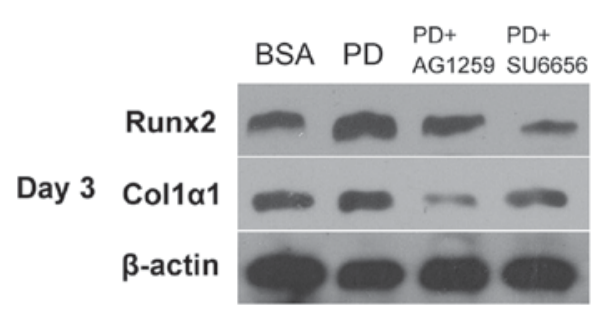

C

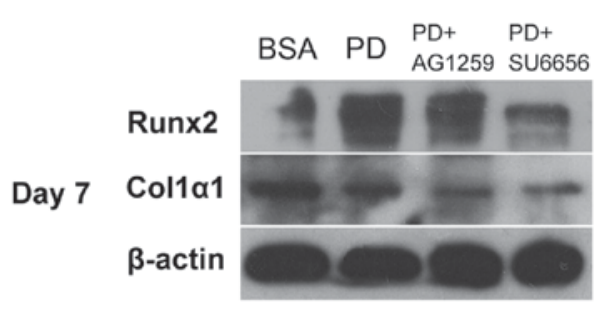

B

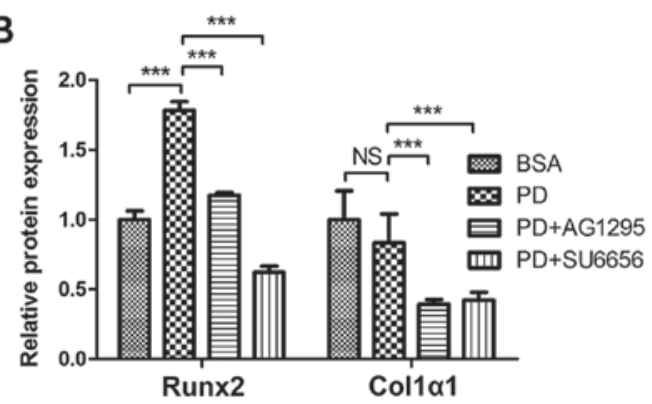

D

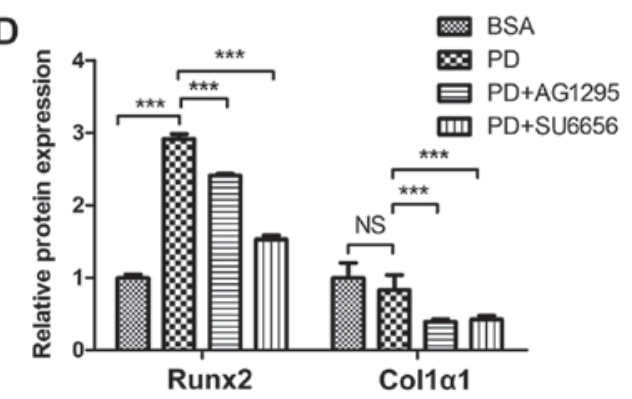

Figure 4. PDGF-BB promotes osteogenic relative protein expression via the Src/JAK2 signaling pathway. (A) MC3T3-E1 cells were treated with BSA ( $25 \mathrm{ng} / \mathrm{ml})$, PDGF-BB $(25 \mathrm{ng} / \mathrm{ml})$, PDGF-BB $(25 \mathrm{ng} / \mathrm{ml})+$ AG1295 $(20 \mu \mathrm{mol} / \mathrm{l})$ and PDGF-BB $(25 \mathrm{ng} / \mathrm{ml})+$ SU6656 $(2 \mu$ mol $/ \mathrm{l})$, in osteogenic media for 3 days or (C) 7 days. (B and D) Densitometric analysis of the associated bands were expressed as the relative optical density of the bands, corrected using $\beta$-actin as the control (normalization bands). Data are presented as the mean \pm standard deviation $(\mathrm{n}=3){ }^{*}{ }^{* * *} \mathrm{P}<0.001$, as indicated. PDGF-BB, platelet-derived growth factor-BB; JAK2, Janus kinase 2; NS, not significant; BSA, bovine serum albumin; Runx2, Runt-related transcription factor 2; Col1 $\alpha 1$, collagen type 1 $\alpha 1$.

A
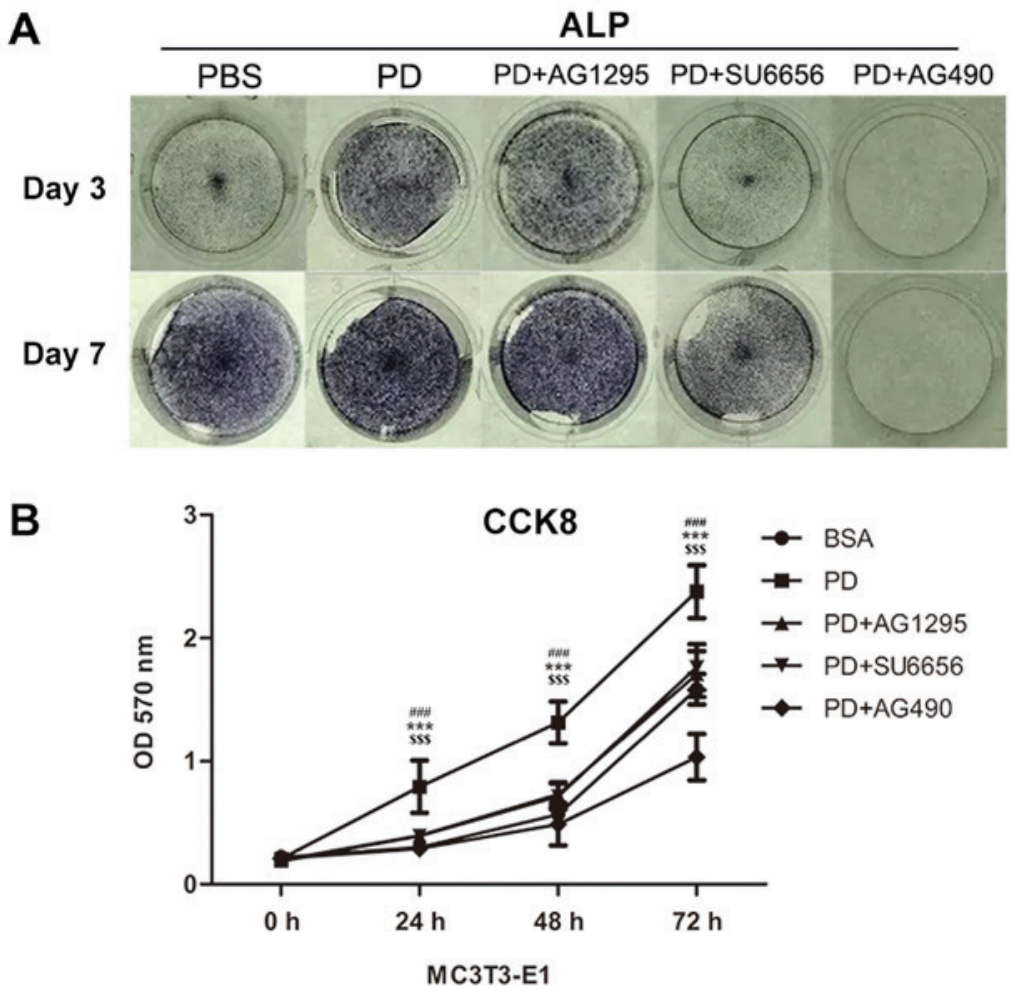

Figure 5. PDGF-BB promotes MC3T3-E1 cell differentiation and proliferation via the Src/JAK2 signaling pathway. (A) Images of M3T3-E1 cells treated with BSA (25 ng/ml), PDGF-BB (25 ng/ml), PDGF-BB (25 ng/ml)+AG1295 $(20 \mu \mathrm{mol} / \mathrm{l})$, PDGF-BB (25 ng/ml)+SU6656 (2 $\mu \mathrm{mol} / \mathrm{l})$ and PDGF-BB $(25 \mathrm{ng} / \mathrm{ml})+\mathrm{AG} 490(10 \mu \mathrm{mol} / \mathrm{l})$ in osteogenic media stained by alkaline phosphatase at 3 and 7 days. (B) The OD values at $450 \mathrm{~nm}$ were then measured for $72 \mathrm{~h}$, and the data were expressed as the mean \pm standard deviation $(\mathrm{n}=3)$ with six replicates. ${ }^{* * *} \mathrm{P}<0.001$ vs. PDGF-BB+AG1295; \#\#" $\mathrm{P}<0.001$ vs. PDGF-BB+SU6656; ${ }^{\$ \$ \$} \mathrm{P}<0.001$ vs. PDGF-BB+AG490. PDGF-BB, platelet-derived growth factor-BB; BSA, bovine serum albumin; JAK2, Janus kinase 2; OD, optical density; CCK, Cell Counting kit; ALP, alkaline phosphatase.

of these genes increased 2-5 fold. In addition, western blot analysis indicated an increase in Runx 2 and Coll $\alpha 1$ at 3 days
(Fig. 4A and B) and 7 days (Fig. 4C and D) in the PDGF-BB group compared with the other groups. A significantly enhanced 
A Alizarin red staining

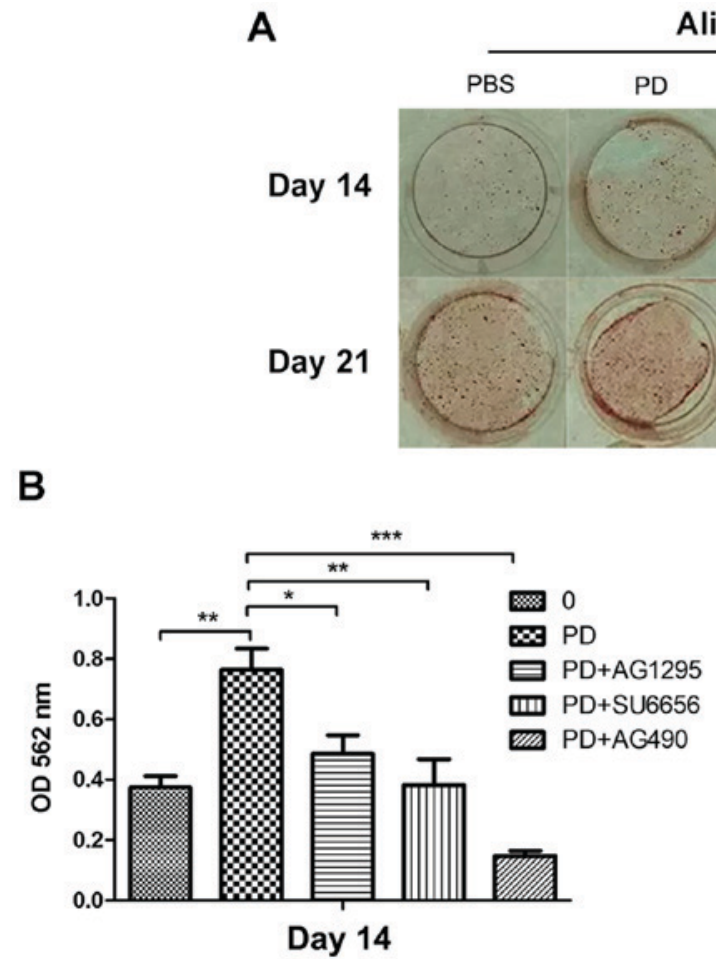

PD+AG1295 PD+SU6656 PD+AG490

Day 14

Day 21

B Day 14

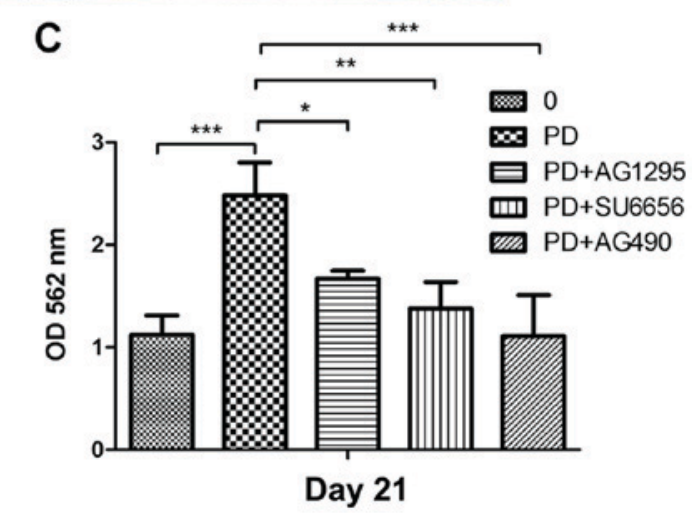

Figure 6. PDGF-BB promotes MC3T3-E1 cell differentiation and mineralization via the Src/JAK2 signaling pathway. (A) M3T3-E1 cells were treated with BSA (25 ng/ml), PDGF-BB (25 ng/ml), PDGF-BB (25 ng/ml)+AG1295 (20 $\mu \mathrm{mol} / \mathrm{l})$, PDGF-BB ( $25 \mathrm{ng} / \mathrm{ml})+$ SU6656 ( $\mu$ mol/l) and PDGF-BB (25 ng/ml)+AG490 $(10 \mu \mathrm{mol} / \mathrm{l})$ in osteogenic media and stained with Alizarin Red at (B) 14 and (D) 21 days. (B and C) Quantification of Alizarin Red S staining with Hexadecylpyridinium Chloride Monohydrate; the amount of released dye was quantified by thermoMUltiskan EX plate reader at $562 \mathrm{~nm}$. "P<0.05, ${ }^{* *} \mathrm{P}<0.01$ and ${ }^{* * *} \mathrm{P}<0.001$, as indicated. PDGF-BB, platelet-derived growth factor-BB; JAK2, Janus kinase 2; OD, optical density; BSA, bovine serum albumin.

ALP signal was observed in the PDGF-BB group compared with the control, PDGF-BB + AG1295, PDGF-BB + SU6656 and PDGF-BB +AG490 groups at 3 and 7 days (Fig. 5A). The Alizarin Red staining intensity was greatly enhanced in the PGDF-BB group compared with the other groups at 14 and 21 days (Fig. 6A-C).

PDGF-BB promotes the proliferation of MC3T3-E1 cells via the $\operatorname{Src} / J A K 2$ signaling pathway. It was not previously determined whether PDGF-BB promotes the proliferation of MC3T3-E1 cells through the Src/JAK2 signaling pathway. Thus, the proliferation rate of cultured MC3T3-E1 cells was quantified with a CCK-8 assay in the present study. It was identified that the OD570 was markedly increased in the PDGF-BB group compared with the control, PDGF-BB + AG1295, PDGF-BB + SU6656 and PDGF-BB + AG490 groups (Fig. 5B).

\section{Discussion}

The association between PDGF-BB and bone formation has been widely studied. PDGF-BB serves a function in osteoblast migration, proliferation and differentiation $(4,6,8,16)$. Previous studies have indicated that PDGF-BB is associated with the apoptosis resistance of chondrocytes through the Src/PI3K/AKT signaling pathway (17) and that PDGF-BB induces metanephric mesenchymal cell migration by activating Src (18). In addition, PDGF-BB promotes pancreatic cancer cell proliferation through the JAK2-STAT3 signaling pathway (7). This indicates that there are interactions between PDGF-BB, Src and JAK2.
In the present study, it was determined whether PDGFR $\beta$, Src and JAK2 were activated in MC3T3-E1 cells following PDGF-BB treatment. The results indicated that PDGF-BB induced the phosphorylation of PDGFR $\beta$, Src and JAK2 in a time-dependent manner. Next, it was examined whether Src was upstream of JAK2. To evaluate this, AG1295, SU6656 and AG490, specific inhibitors of PDGFR $\beta$, Src and JAK2, respectively, were applied. It was identified that SU6656, a specific inhibitor of Src, also suppressed JAK2. However, AG490, a specific inhibitor of JAK2, did not inhibit Src. These inhibitors were also used to determine whether PDGF-BB could promote MC3T3-E1 cell differentiation and proliferation through the Src/JAK2 signaling pathway. Subsequently, it was demonstrated that SU6656 suppressed the activity of Src and JAK2, while AG490 had no effect on Src. Therefore, we hypothesized that Src is upstream of JAK2, which is consistent with a previous study, in which it was demonstrated that c-Src is an upstream molecule mediating thrombin-induced JAK2 and STAT3 activation in WI-38 cells, by the transfection of cells with c-SrcDN (19).

There are conflicting reports regarding the ability of PDGF-BB to promote osteogenesis. A number of studies have indicated that PDGF-BB inhibits the osteogenic differentiation of mesenchymal stem cells by regulating miRNA-138 (20), while others have reported that PDGF-BB could promote host cell migration into artificial bones without inhibiting osteoblastogenesis (21) and enhance DNA synthesis in MC3T3-E1 cells (22). In order to evaluate the potential role of the Src/JAK2 signaling pathway in PDGF-BB-induced osteogenesis, the effect of PDGF-BB on the differentiation and mineralization of MC3T3-E1 cells 
was evaluated. The results of qPCR indicated that PDGF-BB increased the expression of osteogenic genes, including Runx2, Osterix, ALP, OCN, OPN and Col1 $\alpha 1$. In addition, SU6656, an inhibitor of Src, significantly reduced the effect of PDGF-BB on these genes. The western blot analysis of Runx 2 and Col1 $\alpha 1$ expression supported these results. These findings indicated that PDGF-BB increases the expression of osteogenic genes and proteins, and that Src and JAK2 are involved in the process. Consistent with these results, it was also identified that PDGF-BB enhanced ALP staining. This effect was reduced by the suppression of Src or JAK2 signaling pathway, with similar results for Alizarin Red staining. These data suggest that PDGF-BB promotes the osteogenic differentiation of MC3T3-E1 cells, and that the Src/JAK2 signaling pathway was involved in this process.

As PDGF-BB was reported to promote the proliferation of human osteoblasts (4), the effects and potential mechanisms of PDGF-BB on MC3T3-E1 cells were investigated in the current study. The results indicated that PDGF-BB promoted MC3T3-E1 cell proliferation compared with the control and inhibitor treatment groups. This suggested that suppressing the Src/JAK2 signaling pathway also suppressed the PDGF-BB induction of MC3T3-E1 cell proliferation. It was therefore hypothesized that the Src/JAK2 signaling pathway may be involved in the PDGF-BB-induced increase in MC3T3-E1 cell proliferation.

In addition to its effects on bone formation and regeneration $(6,23)$, the ability of PDGF-BB to promote migration has also been extensively studied in osteoblasts $(16,24)$ and epithelial mesenchymal cells (25). Numerous studies have indicated that PDGF-BB may act on vascular smooth muscle cells, as an example, to promote migration $(16,26)$ and proliferation $(27,28)$, and ability of PDGF-BB to induce angiogenesis has also been demonstrated $(8,29-32)$. Therefore, PDGF-BB may serve a central function in the healing of bone fractures, as it can not only induce osteoblast differentiation, a key factor in osteogenesis, but also vascular smooth muscle cell migration and angiogenesis, which is critical for the survival of new bone. This illustrates the important function of PDGF-BB in osteogenesis and the necessity of further research into the mechanism of PDGF-BB in inducing MC3T3-E1 cell differentiation and proliferation.

In summary, the present study demonstrated that PDGF-BB could promote MC3T3-E1 cell differentiation and proliferation through the Src/JAK2 signaling pathway. This suggests that PDGF-BB could be an important bone regulatory factor and provides a better understanding into the molecular mechanisms of MC3T3-E1 cells. These results may inform a novel strategy for the treatment of patients with bone fractures or osteoporosis.

\section{Acknowledgements}

Not applicable.

\section{Funding}

The present study was supported by National Natural Science Foundation of China (grant nos. 81470718 and 81771051) for the design of the study, data collection and analysis, and manuscript writing; and by Hubei Province Health and Family
Planning Scientific Research Project (grant no. WJ2017M046)

for data collection, analysis and interpretation.

\section{Availability of data and materials}

The datasets used and analyzed during the current study are available from the corresponding author on reasonable request.

\section{Authors' contributions}

YZ cultured the cells, and collected, analyzed and interpreted the data from the cell proliferation assay, histology test and RT-qPCR. QL performed the western blotting and analyzed the data, and was a major contributor in writing the manuscript. ZL conceived, designed and supervised the study. All authors read and approved the final manuscript.

\section{Ethics approval and consent to participate}

The application of the cell line MC3T3-E1 used in the present study was approved by the Ethics Committee of School and Hospital of Stomatology, Wuhan University (Hubei, China).

\section{Patient consent for publication}

Not applicable.

\section{Competing interests}

The authors declare that they have no competing interests.

\section{References}

1. Li J: JAK-STAT and bone metabolism. JAKSTAT 2: e23930, 2013.

2. Luo J, Yang Z, Ma Y, Yue Z, Lin H, Qu G, Huang J, Dai W, Li C, Zheng C, et al: LGR4 is a receptor for RANKL and negatively regulates osteoclast differentiation and bone resorption. Nat Med 22: 539-546, 2016.

3. Li CH, Zhao JX, Sun L, Yao ZQ, Deng XL, Liu R and Liu XY: AG490 inhibits NFATc1 expression and STAT3 activation during RANKL induced osteoclastogenesis. Biochem Biophys Res Commun 435: 533-539, 2013.

4. Vordemvenne T, Paletta JR, Hartensuer R, Pap T, Raschke MJ and Ochman S: Cooperative effects in differentiation and proliferation between PDGF-BB and matrix derived synthetic peptides in human osteoblasts. BMC Musculoskelet Disord 12: 263, 2011.

5. Takai S, Matsushima-Nishiwaki R, Adachi S, Natsume H, Minamitani C, Mizutani J, Otsuka T, Tokuda H and Kozawa O: (-)-Epigallocatechin gallate reduces platelet-derived growth factor-BB-stimulated interleukin- 6 synthesis in osteoblasts: Suppression of SAPK/JNK. Mediators Inflamm 2008: 291808, 2008.

6. Caplan AI and Correa D: PDGF in bone formation and regeneration: New insights into a novel mechanism involving MSCs. J Orthop Res 29: 1795-1803, 2011.

7. Masamune A, Satoh M, Kikuta K, Suzuki N and Shimosegawa T: Activation of JAK-STAT pathway is required for platelet-derived growth factor-induced proliferation of pancreatic stellate cells. World J Gastroenterol 11: 3385-3391, 2005.

8. Xie H, Cui Z, Wang L, Xia Z, Hu Y, Xian L, Li C, Xie L, Crane J, Wan M, et al: PDGF-BB secreted by preosteoclasts induces angiogenesis during coupling with osteogenesis. Nat Med 20: 1270-1278, 2014.

9. Lennartsson J, Ma H, Wardega P, Pelka K, Engström U, Hellberg $\mathrm{C}$ and Heldin $\mathrm{CH}$ : The Fer tyrosine kinase is important for platelet-derived growth factor-BB-induced signal transducer and activator of transcription 3 (STAT3) protein phosphorylation, colony formation in soft agar, and tumor growth in vivo. J Biol Chem 288: 15736-15744, 2013. 
10. Rucci N, Susa M and Teti A: Inhibition of protein kinase c-Src as a therapeutic approach for cancer and bone metastases. Anticancer Agents Med Chem 8: 342-349, 2008.

11. Ishizawar R and Parsons SJ: c-Src and cooperating partners in human cancer. Cancer Cell 6: 209-214, 2004.

12. Waibel M, Solomon VS, Knight DA, Ralli RA, Kim SK, Banks KM, Vidacs E, Virely C, Sia KC, Bracken LS, et al: Combined targeting of JAK2 and Bcl-2/Bcl-xL to cure mutant JAK2-driven malignancies and overcome acquired resistance to JAK2 inhibitors. Cell Rep 5: 1047-1059, 2013.

13. Mori K, Blanchard F, Charrier C, Battaglia S, Ando K, Duplomb L, Shultz LD, Redini F and Heymann D: Conditioned media from mouse osteosarcoma cells promote MC3T3-E1 cell proliferation using JAKs and PI3-K/Akt signal crosstalk. Cancer Sci 99: 2170-2176, 2008.

14. Ge X, Chen SY, Liu M, Liang TM and Liu C: Evodiamine inhibits PDGF-BB-induced proliferation of rat vascular smooth muscle cells through the suppression of cell cycle progression and oxidative stress. Mol Med Rep 14: 4551-4558, 2016.

15. Wei J, Shimazu J, Makinistoglu MP, Maurizi A, Kajimura D, Zong H, Takarada T, Lezaki T, Pessin JE, Hinoi E and Karsenty G: Glucose uptake and Runx2 synergize to orchestrate osteoblast differentiation and bone formation. Cell 161: 1576-1591, 2015.

16. Colciago A, Celotti F, Casati L, Giancola R, Castano SM, Antonini G, Sacchi MC and Negri-Cesi P: In vitro effects of PDGF isoforms (AA, BB, $\mathrm{AB}$ and $\mathrm{CC}$ ) on migration and proliferation of SaOS-2 osteoblasts and on migration of human osteoblasts. Int J Biomed Sci 5: 380-389, 2009.

17. Montaseri A, Busch F, Mobasheri A, Buhrmann C, Aldinger C, Rad JS and Shakibaei M: IGF-1 and PDGF-bb suppress IL-1 $\beta$-induced cartilage degradation through down-regulation of NF-кB signaling: Involvement of Src/PI-3K/AKT pathway. PLoS One 6: e28663, 2011.

18. Wagner B and Gorin Y: Src tyrosine kinase mediates platelet-derived growth factor BB-induced and redox-dependent migration in metanephric mesenchymal cells. Am J Physiol Renal Physiol 306: F85-F97, 2014.

19. Bai KJ, Chen BC, Pai HC, Weng CM, Yu CC, Hsu MJ, Yu MC, Ma HP, Wu CH, Hong CY, et al: Thrombin-induced CCN2 expression in human lung fibroblasts requires the c-Src/JAK2/STAT3 pathway. J Leukoc Biol 93: 101-112, 2013.

20. Qu B, Xia X, Wu HH, Tu CQ and Pan X: PDGF-regulated miRNA-138 inhibits the osteogenic differentiation of mesenchymal stem cells. Biochem Biophys Res Commun 448: 241-247, 2014.

21. Yoshida S, Iwasaki R, Kawana H, Miyauchi Y, Hoshi H, Miyamoto H, Mori T, Kanagawa H, Katsuyama E, Fujie A, et al: PDGFBB promotes PDGFRa-positive cell migration into artificial bone in vivo. Biochem Biophys Res Commun 421: 785-789, 2012.
22. Tsukamoto T, Matsui T, Fukase M and Fujita T: Platelet-derived growth factor B chain homodimer enhances chemotaxis and DNA synthesis in normal osteoblast-like cells (MC3T3-E1). Biochem Biophys Res Commun 175: 745-751, 1991.

23. Chen W, Baylink DJ, Brier-Jones J, Neises A, Kiroyan JB, Rundle $\mathrm{CH}$, Lau KH and Zhang XB: PDGFB-based stem cell gene therapy increases bone strength in the mouse. Proc Natl Acad Sci USA 112: E3893-E3900, 2015.

24. Hengartner NE, Fiedler J, Ignatius A and Brenner RE: IL-1 $\beta$ inhibits human osteoblast migration. Mol Med 19: 36-42, 2013.

25. Yang L, Lin C and Liu ZR: P68 RNA helicase mediates PDGF-induced epithelial mesenchymal transition by displacing Axin from beta-catenin. Cell 127: 139-155, 2006.

26. Kang H, Ahn DH, Pak JH, Seo KH, Baek NI and Jang SW Magnobovatol inhibits smooth muscle cell migration by suppressing PDGF-R $\beta$ phosphorylation and inhibiting matrix metalloproteinase-2 expression. Int J Mol Med 37: 1239-1246, 2016.

27. Park S, Kim JK, Oh CJ, Choi SH, Jeon JH and Lee IK: Scoparone interferes with STAT3-induced proliferation of vascular smooth muscle cells. Exp Mol Med 47: e145, 2015.

28. Yan G, Wang Q, Hu S, Wang D, Qiao Y, Ma G, Tang C and Gu Y: Digoxin inhibits PDGF-BB-induced VSMC proliferation and migration through an increase in ILK signaling and attenuates neointima formation following carotid injury. Int J Mol Med 36: 1001-1011, 2015.

29. Rykala J, Przybylowska K, Majsterek I, Pasz-Walczak G, Sygut A, Dziki A and Kruk-Jeromin J: Angiogenesis markers quantification in breast cancer and their correlation with clinicopathological prognostic variables. Pathol Oncol Res 17: 809-817, 2011.

30. Drela E, Kulwas A, Jundziłł W, Góralczyk B, Boinska J, Drewniak W, Gadomska G and Rość D: VEGF-A and PDGF-BB-angiogenic factors and the stage of diabetic foot syndrome advancement. Endokrynol Pol 65: 306-312, 2014.

31. Han H, Cao FL, Wang BZ, Mu XR, Li GY and Wang XW: Expression of angiogenesis regulatory proteins and epithelial-mesenchymal transition factors in platelets of the breast cancer patients. ScientificWorldJournal 2014: 878209, 2014.

32. Yin T,He S, Su C, Chen X, Zhang D, Wan Y, Ye T, Shen G, Wang Y, Shi $\mathrm{H}$, et al: Genetically modified human placenta-derived mesenchymal stem cells with FGF-2 and PDGF-BB enhance neovascularization in a model of hindlimb ischemia. Mol Med Rep 12: 5093-5099, 2015

This work is licensed under a Creative Commons Attribution-NonCommercial-NoDerivatives 4.0 International (CC BY-NC-ND 4.0) License. 